\title{
Polyvalent Vaccine
}

National Cancer Institute

\section{Source}

National Cancer Institute. Polyvalent Vaccine. NCI Thesaurus. Code C2848.

A vaccine designed to elicit an immune response either to more than one infectious agent or to several different antigenic determinants of a single agent. 